\title{
Nanomechanical Measurements in Harsh Environments
}

\author{
PETER HOSEMANN ${ }^{1,3}$ and XIAODONG $\mathrm{LI}^{2,4}$ \\ 1.-Department of Nuclear Engineering, University of California, Berkeley, USA. 2.-Department \\ of Mechanical and Aerospace Engineering, University of Virginia, Charlottesville, USA. \\ 3.—e-mail: peterh@berkeley.edu. 4.—e-mail: xl3p@eservices.virginia.edu
}

Materials properties are size and environment dependent. Nanomechanical measurement techniques in ambient environments have been well established. However, how materials properties change with external harsh environments is barely quantified or understood. Many materials and devices are fabricated and/or used in harsh environments. For instance, sensors and actuators are normally exposed to high temperatures, chemical/ biological species, and irradiation. The electrodes in energy conversion and storage systems are immersed into chemical electrolytes to be functional. Electron-beam lithography, chemical etching, and/or ion-beam etching are essential steps in the ultra-large-scale integration of nanostructures to fabricate micro/nanodevices. Gaining such knowledge is crucial to design robust systems for harsh environments, avoiding disastrous failure accidents and improving the safety of facilities and devices.

This collection of papers focuses on "Nanomechanical Measurements in Harsh Environments" in science and research, as well as on emerging engineering applications. The importance of probing local mechanical properties in the intended environment, be it high temperature, radiation, or corrosive, is outlined in a number of studies in this topic. Several outstanding publications are presented from a range of international scientists discussing how these difficult measurements are carried out. These publications can foster new knowledge in this area of research.

How materials react to extreme temperatures is of particular interest. S. Bigl discusses how amorphous silicon dioxide films used as dielectric layers in microelectronic devices have seen crack formation due to hydrogen and nitrogen being incorporated into the films at the annealing temperature of

Peter Hosemann and Xiaodong $\mathrm{Li}$ are the guest editors for the Nanomechanical Materials Behavior Committee of the TMS Materials Processing \& Manufacturing Division and coordinators of the topic "Nanomechanical Measurements in Harsh Environments" in this issue. $1000^{\circ} \mathrm{C}$. However, it is revealed that in the past, tests were done only to a temperature of $500^{\circ} \mathrm{C}$, so previous methods could not capture the complete thermomechanical behavior of the films. Bigl shows that, by doing in situ film stress measurements using a wafer curvature technique, results can be collected at the actual annealing temperature. This resulted in the discovery that at temperatures above $550^{\circ} \mathrm{C}$, there were increases in stress and a change in film chemistry, which were causing the crack formations.

Another work devoted to the evolution of micromechanical behavior with temperature was written by Wheeler. This investigation was done to understand the mechanical properties near transformation temperatures of precipitation-hardenable $\mathrm{Ni}$ 48Ti-25Pd shape memory alloy. Micro-compression samples were tested in-situ in the scanning electron microscope (SEM). These samples were brought to a temperature near the martensite and austenite phase transformation region. The material had stable pseudoelastic behavior, and in the plastic region, slipping of the fine precipitates was observed.

Developing new techniques to obtain micro-scale mechanical properties in extreme temperature environments has been the subject of many recent works, and in Armstrong's article, a new method called micro-scale bend testing geometry was proposed to find the elastic, plastic, and fracture behavior of silicon at around $770^{\circ} \mathrm{C}$. These results were then checked by the widely accepted micropillar compression method. Armstrong was able to determine how the Young's modulus of the material decreased and how the material failed as the temperature was increased.

The point at which materials fail under continuously harsh and corrosive conditions is also of great interest. Bumgardner's paper looks at the thermal coatings used on the turbines in jet engines. A nickel alloy substrate with a zirconia-based coating was cyclically worn to imitate the harsh temperature and pressure environment experienced by a 
turbine blade. By using a method of contact models and spectroscopy images, Baumgardner was able to find the maximum shear and its corresponding depth, the time and location the thermal barrier separated from the substrate, and the root causes of the separation.

Thermal drift in time-dependent studies, such as long-term creep, can introduce great sources of error into the results of those studies. As such, the characterization of the change of material properties when exposed to a range of temperatures is of importance. V. Maier tested fused quartz and ultrafine-grained $\mathrm{Au}$ by nanoindention over a varied range of temperatures and depths. The paper concludes that thermal drift is such a source of error that the data are useful only after being corrected by dynamic stiffness measurements taken during the experiments. However, for the corrected ultrafine-grained $\mathrm{Au}$, it was noticed that there were significant changes in the elastic and plastic properties after the sample reached $200^{\circ} \mathrm{C}$.

Irradiation can sometimes alter the mechanical properties of a material. J. Marsh's paper discusses zirconium alloys that are typically used for nuclear purposes. Marsh's article tests several variables of the alloys, including temperature, manufacturing processes such as annealing and rolling, and levels of irradiation. The study was done for rolled and rolled-annealed samples to accomplish two different microstructures. Nanoindention was done on the samples during the varied ranges of temperatures and irradiation levels to determine the changes in material properties. It was found that the irradiation-induced hardness of the material was more significant in the rolled sample than the rolled-annealed sample and that the yield strength of the irradiated material was higher than the nonirradiated samples.

Another study discussing radiation damage was performed by Vo et al. In this study, micro-compression testing and nanoindentation was carried out on 304L stainless steel before and after ion beam irradiation. It was found that the ion beam irradiation causes a yield strength and hardness increase of the material and that this property change persists even at elevated temperature testing. Performing the post-irradiation characterization over a wide temperature range all the way to light water reactor operating temperature allows for learning more about the deformation behavior of this widely used material in service. In addition to evaluating nanohardness and critical resolved shear stress, it was found that significantly larger and more pronounced slip steps can be observed on the highly irradiated micro-pillars over all the temperature ranges measured. This work is the first time microcompression testing has been carried out on irradiated materials over reactor operating temperatures.

The JOM staff and guest editors hope that these articles introduce the potential of several novel harsh-environment nanomechanical testing methods to a broad JOM audience, leading to further advances in nanomechanical testing instrumentation, exploration of the nanomechanical behavior of materials, and a fundamental understanding of size effects.

The following papers being published under the topic of "Nanomechanical Measurements in Harsh Environments" provide excellent details and research on the subject. To download any of the papers, go to the following url: http://link.springer.com/journal/ $11837 / 67 / 12 /$ page/ 1 to the table of contents page for the December 2015 issue (vol. 67, no. 12):

- "High-Temperature Characterization of Silicon Dioxide Films with Wafer Curvature," S. Bigl, W. Heinz, M. Kahn, H. Schoenherr, and M.J. Cordill

- "Elevated Temperature, In Situ Micromechanical Characterization of a High-Temperature Ternary Shape-Memory Alloy," J.M. Wheeler, C. Niederberger, R. Raghavan, G. Thompson, M. Weaver, and J. Michler

- "Bend Testing of Silicon Microcantilevers from $21^{\circ} \mathrm{C}$ to $770^{\circ} \mathrm{C}$," David E.J. Armstrong and Edmund Tarleton

- "Unveiling Ultra-High Temperature Wear and Indentation Damage Mechanisms of Thermal Barrier Coatings," Clifton Bumgardner and Xiaodong $\mathrm{Li}$

- "Thermally Activated Deformation Behavior of ufg-Au: Environmental Issues During LongTerm and High-Temperature Nanoindentation Testing," Verena Maier, Alexander Leitner, Reinhard Pippan, and Daniel Kiener

- "Nanomechanical Characterization of Temperature-Dependent Mechanical Properties of IonIrradiated Zirconium with Consideration of Microstructure and Surface Damage," Jonathan Marsh, Yang Zhang, Devendra Verma, Sudipta Biswas, Aman Haque, and Vikas Tomar

- "Small-Scale Mechanical Testing on Proton Beam-Irradiated 304 SS from Room Temperature to Reactor Operation Temperature," H. Vo, A. Reichardt, C. Howard, M.D. Abad, D. Kaoumi, P. Chou, and P. Hosemann 